www.jmscr.igmpublication.org

Impact Factor (SJIF): 6.379

Index Copernicus Value: 71.58

ISSN (e)-2347-176x ISSN (p) 2455-0450

crossrefDOI: https://dx.doi.org/10.18535/jmscr/v6i8.12

\begin{abstract}
Journal Of Medical Science And Clinical Research
IGM Publication

An Official Publication of IGM Publication
\end{abstract}

\title{
Original Research Article \\ Lip Leishmaniasis: A New Emerging Clinical form of Cutaneous Leishmaniasis from sub Himalayan Region
}

\author{
Authors \\ Dr Sandhya Kumari ${ }^{1}$, Dr Ashok Garg ${ }^{2 *}$ \\ ${ }^{1}$ Dermatologist, Indira Gandhi Medical College Shimla (H.P.) \\ ${ }^{2}$ Pediatrician, MGMSC Khaneri, Rampur, Shimla (H.P.) \\ *Corresponding Author \\ Dr Ashok Garg \\ Address: House no. 35, Ward no. 2, Village Anu kalan, PO/Teh/ Distt. Hamirpur (HP)-177001, India \\ Email: ashokgarg44@gmail.com, 9459930003, 8894903002
}

\begin{abstract}
Background: Muco-cutaneous leishmaniasis (MCL) is a disease of new world and characterized by involvement of oral, nasal, pharyngeal, laryngeal and lip mucosae. Mucosal involvement is rarely seen with old world leishmaniasis. Recently, cases of lip mucosal leishmaniasis $(L M L)$ have been reported from old world and they are considered to be a part of cutaneous leishmaniasis (CL) rather than of MCL.

Methods: All newly diagnosed cases of $C L$ were registered and detailed clinico-epidemiological variables were recorded. Data of patients having lesions over lips was extracted out and analysed separately. Imprint smears for Leishmania bodies and biopsies for histopathology were done only in atypical cases to confirm clinical diagnosis.

Results: Out of total 337 registered cases, 73 patients had lesions over lips either as sole presentation or in association with cutaneous lesions. Amongst affected cases of LML no predilection was found for specific gender or age group. Duration of disease varied from 20 days to one year but most [60 (67.3\%)] cases presented with in six month of lesion onset. Although multiple lesions were also present but $42(57.5 \%)$ cases presented with single lesion. Nodules and plaques were the most frequent morphologies found in 28(38.4\%) and 26(35.6\%) cases respectively. Upper lip was most commonly affected site in 43(58.9\%) cases followed by lower lip in 15(20.54\%) and oral commissure in $9(12.33 \%)$ cases. Out 73 cases $21(28.76 \%)$ cases had associated skin lesions and 3(4.1\%) cases had extension of lesions to either oral or nasal mucosa $(M C L)$. Biopsies and imprint smear examination were done in 11(15.06\%) atypical cases. Out of these 11 cases, 8(72.7\%) cases showed features suggestive of leishmaniasis on HPE and only 2(18.18\%) cases showed imprint smear positivity while in rest of the cases histopathology and imprint smears were non-conclusive (excluded out from study). Most cases were treated effectively with intralesional injection of sodium stibogluconate (I/L SSG). But in 3 cases with MCL, intravenous SSG was given together with oral itraconazole to achieve complete cure. Most 69(94.5\%) cases were cured completely after 3rd cycle of I/L SSG, only $3(4.1 \%)$ patients took 4 cycles and 1(1.7\%) patient took 5 cycles for complete resolution of lesion. Immediate side effects like pain, swelling, bleeding and ulceration were observed almost in every patient. Scarring, milia formation and reactive sub-mandibular lymphadenopathy were other less commonly observed side effects.

Conclusion: This is the first study on lip leishmaniasis from Himachal Pradesh. Lip leishmaniasis is not uncommon but very rarely reported entity from our region. Unusual presentation poses diagnostic challenge and we recommend facilities of culture, PCR and molecular studies in order to know about the exact characteristics of parasite and vector in this region. Sodium stibogluconate is the treatment of choice.

Keywords: Lip, Leishmaniasis, Epidemiology, Muco-cutaneous.
\end{abstract}




\section{Introduction}

Leishmaniasis is an infectious disease, caused by Leishmania parasite and transmitted by Phlebotomus sandfly. Depending on the species and the person's immune system, it can manifest into cutaneous leishmaniasis (CL), mucocutaneous leishmaniasis (MCL) and visceral leishmaniasis (VL). ${ }^{[1]}$ CL may appear at unusual sites or present with atypical morphologies. The lip is considered one of the unusual sites with various atypical presentations. ${ }^{[2]}$ Peculiarity of lips is that they act as a gateway between skin and oral mucosa. So CL may involve lips and can progress into MCL. Lip involvement in leishmaniasis may result from direct extension of nearby skin lesions or from hematogenous or lymphatic dissemination of amastigotes from the skin. ${ }^{[4]}$ Generally MCL is a disease of new world and caused by Leishmania braziliensis. Oral and nasal mucosae are commonly affected in MCL while laryngeal and pharyngeal mucosae are rarely involved. ${ }^{[5]} \mathrm{MCL}$ is an important disease and public health problem, because it has a significant morbidity and mortality. ${ }^{[6]}$ Mucosal involvement is rarely seen with old world leishmaniasis. ${ }^{[6]}$ Primary lip mucosal leishmaniasis (LML) is now a common presentation from old world/non- endemic areas. LML as CL not as a part of MCL type has been infrequently mentioned in the medical literature. ${ }^{\text {[7] }}$ LML is important because it can progress into MCL and can cause mutilations, morbidities and mortalities. Further it is a clinical mimicker of many dermatoses like grannulomatous cheilitis, sarcoidosis and actinic cheilitis etc. ${ }^{[8]}$ Being diagnostic and therapeutic challenge for physicians, availability of good diagnostic tests other than conventional investigations are recommended.

\section{Methods}

This prospective observational study was conducted over a period of one year from August 2016 to September 2017. All newly diagnosed cases of cutaneous leishmaniasis (CL) were registered. Demographic profile including age, sex, occupation, locality, family history and history of high risk activities (keeping domestic animals, farming/horticultural activities) were recorded. Clinical details about number, size, site, duration and type of lesion were also noted down. Diagnosis of CL was mainly based on the clinical criteria proposed by Kubba and AI- Gindan. [9] Then clinico-epidemiological data of patients with lip involvement was extracted out and analysed separately. In typical cases treatment was initiated after clinical diagnosis while in atypical/doubtful cases, imprint smears for Leishman Donovan (LD) bodies and tissue smears for histopathology were taken. As facilities for Novy-MacNeal-Nicolle (NNN) culture medium and PCR were not available in this institute so unproven cases were referred to higher centre for further workup. Thus, unconfirmed cases and those patients who lost on follow up examination were excluded out from this study. In all diagnosed cases treatment was given as intralesional sodium stibogluconate injection (I/L SSG) on three alternate days every month (one cycle). Monthly follow up examination was done in every case for treatment response and treatment related minor side effects.

\section{Results}

A total of 337 patients of cutaneous leishmaniasis (CL) were registered over a period of one year. Out of these 337 cases, 73 patients had lesions over lips either as sole presentation or in association with cutaneous lesions. Amongst 73 cases of lip leishmaniasis, male to female ratio was 1.02. (Figure 1) Most cases were seen in age group 31-40 years followed by 0-20 years. (Figure 2) almost all age groups were affected equally with slight lower occurrence after 41 years of age. 


\section{JMSCR Vol||06||Issue||08||Page 62-69||August}

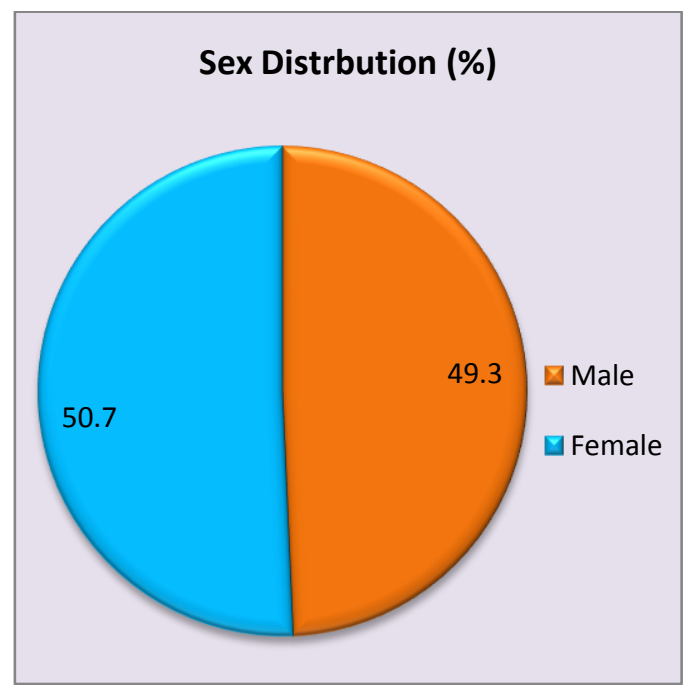

Figure-1

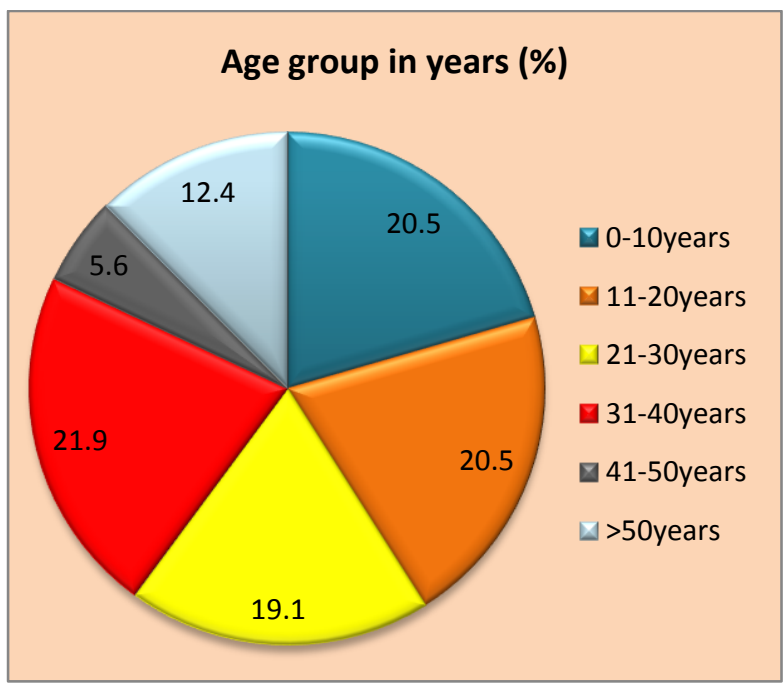

Figure-2

Although males and females were almost equally affected, but it was found that in the age group of 0-10 years (males $=10$, females $=5$ ) and 11-20 the females $(n=5)$ by a ratio of $2: 1$. While after 21 years of age, females were affected more than males in a ratio of 2:1 as shown in the figure 3 . years $($ males $=11$, females $=4$ ), males outnumbered

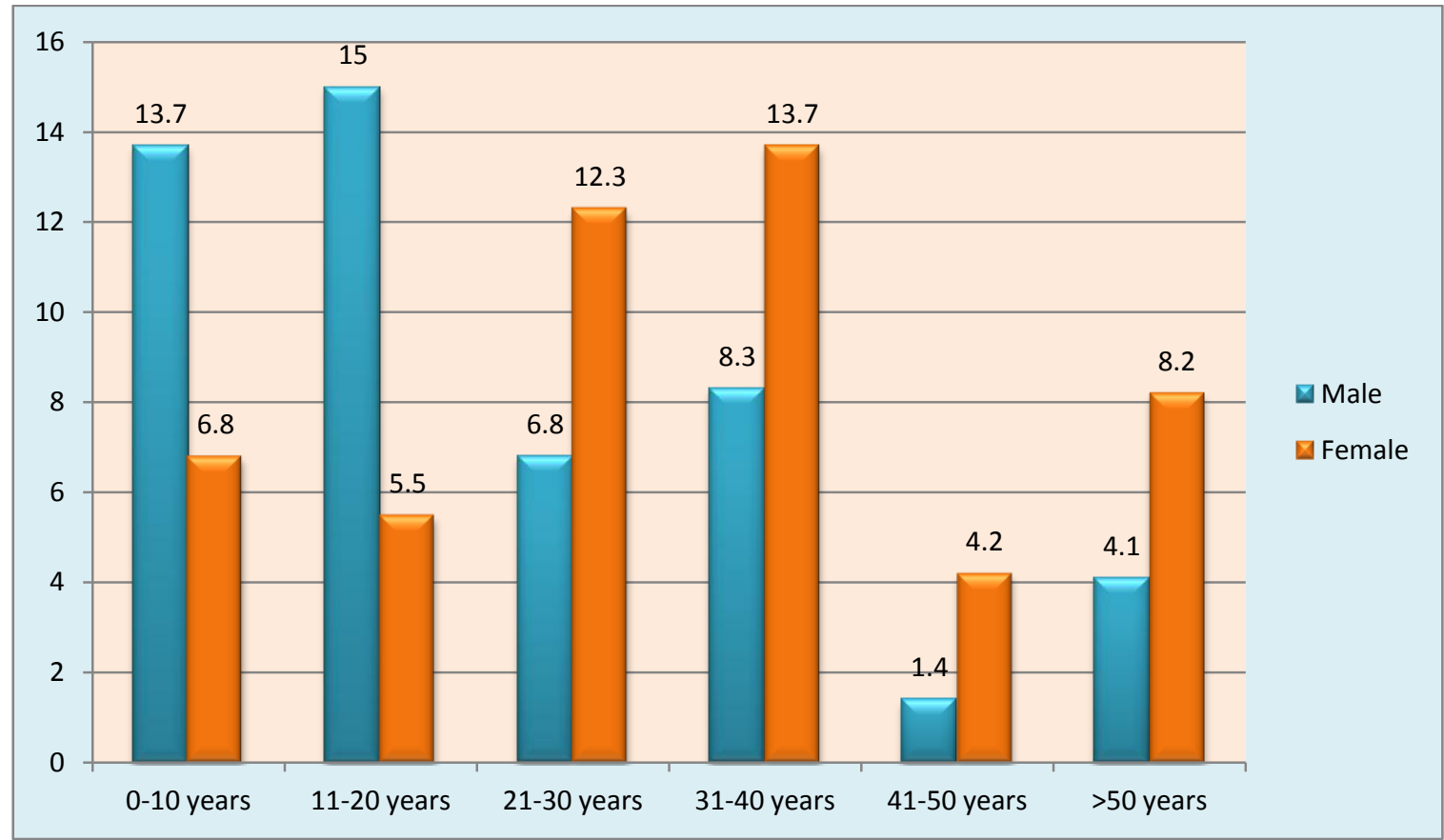

Figure 3: Age and Sex wise distribution of cases with lip leishmaniasis [ $n=73]$

Regional distribution showed maximum cases from endemic foci of district Shimla (Rampur, Sunni block) followed by Kullu (Aani and
Nirmand Block) and Kinnaur (Tapri Block) (Figure 4). 


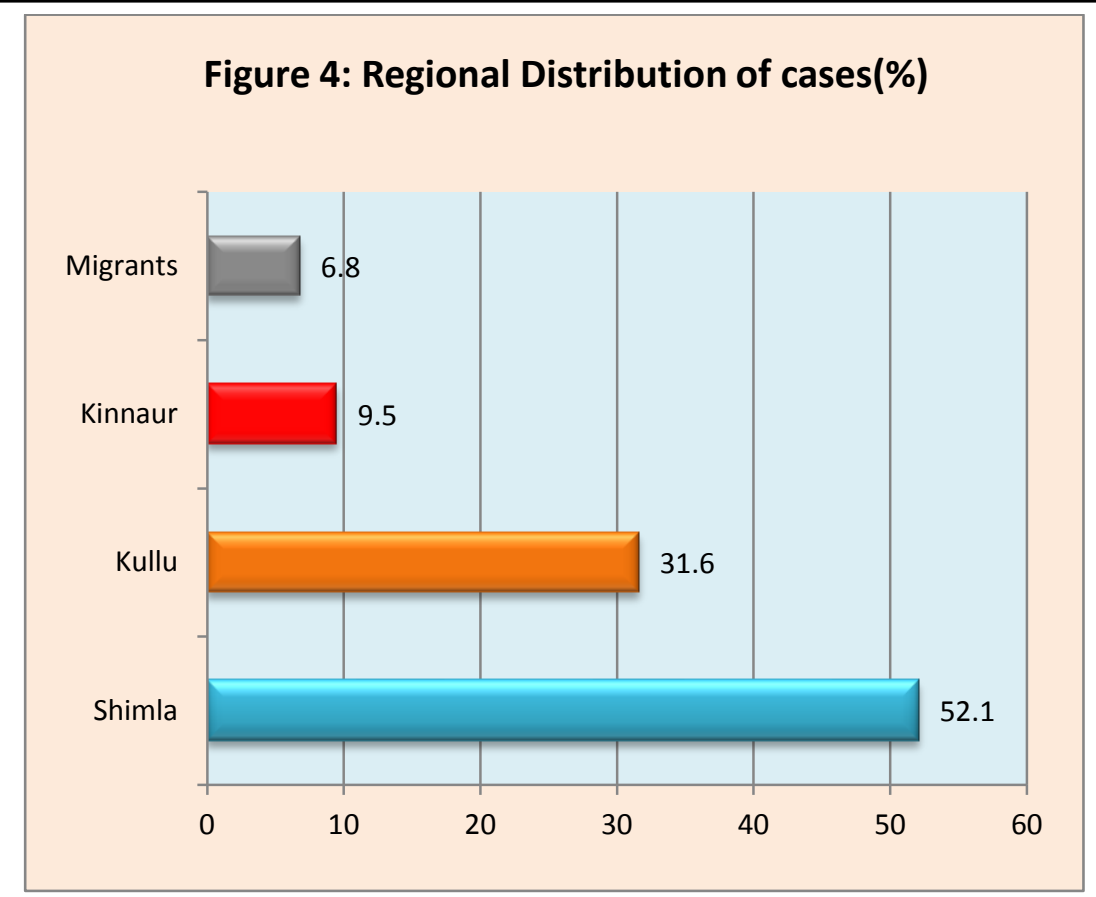

Month wise presentation of cases revealed slight more cases in the month of August and lesser in the months of November and December. (Figure 5)

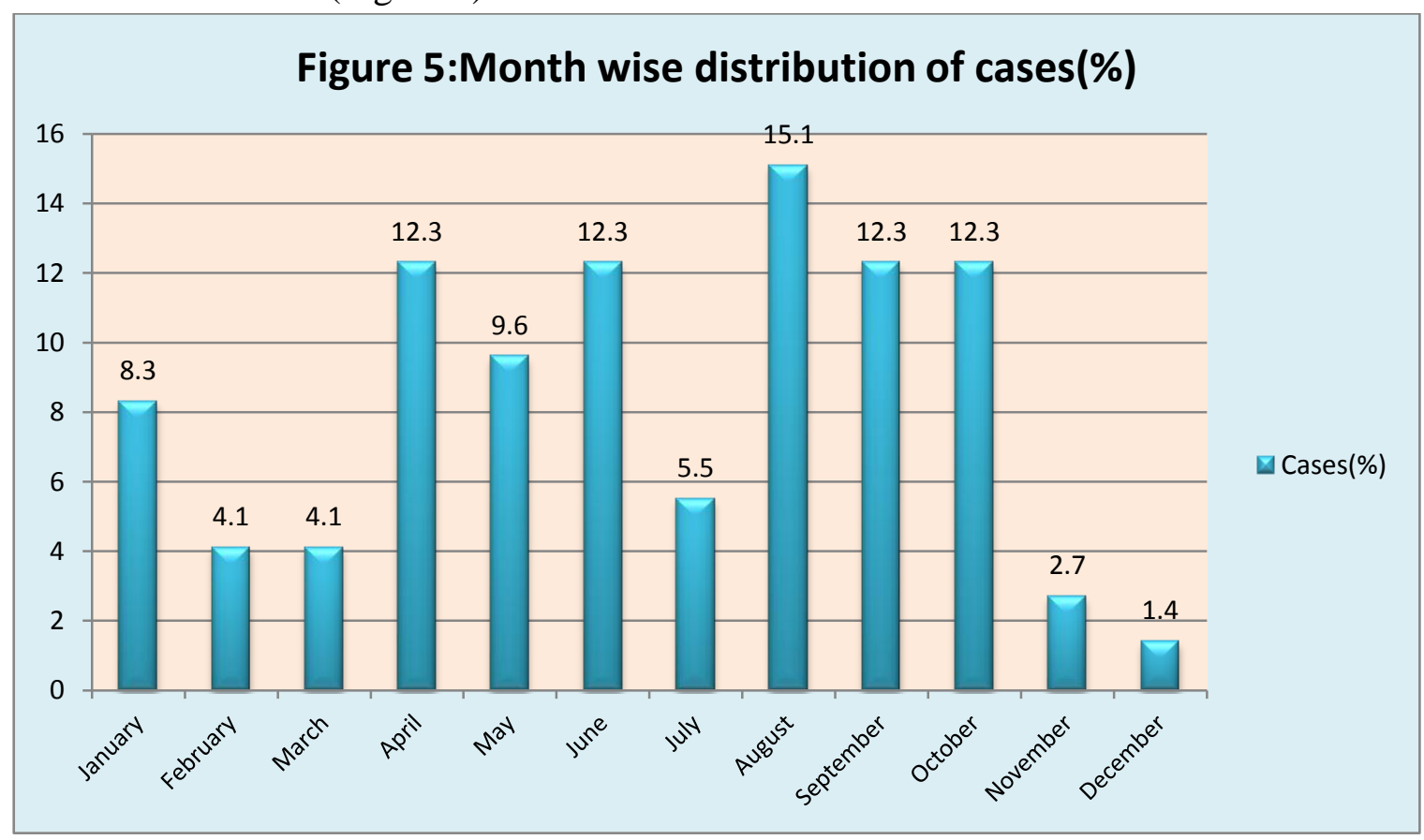

Most 60(67.3\%) cases presented between 1-6 months of lesion onset. Only $3(4.1 \%)$ cases came within one month and 10(13\%) cases came after 6 months of lesion onset. Single lesion was most common presentation in $42(57.5 \%)$ cases and 2 lesions were present in $18(24.7 \%)$ cases. Lip lesions were maximum upto 2 in number in $13(17.8 \%)$ cases, while in rest of the cases (17) multiple lesions were present over extramucocutaneous sites concurrent with lip lesions. 


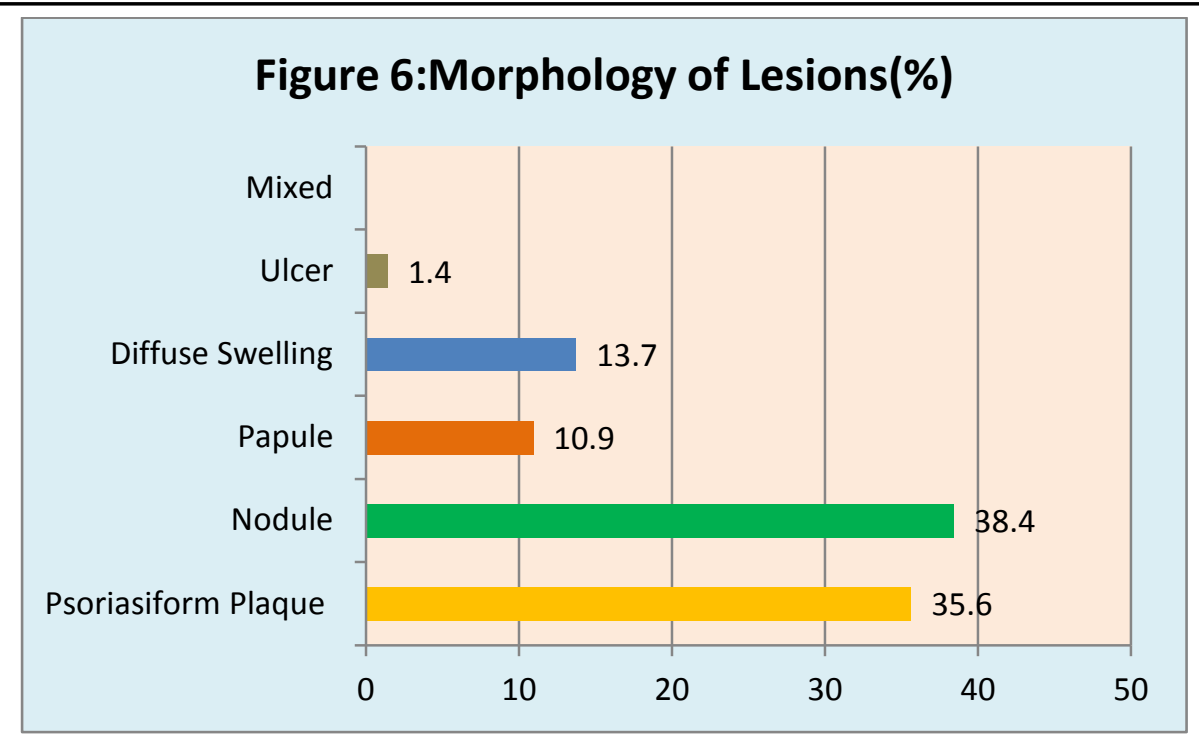

As per morphology, nodules and plaques were most frequent types seen in 28(38.4) and $26(35.6 \%)$ cases respectively. Other variants were

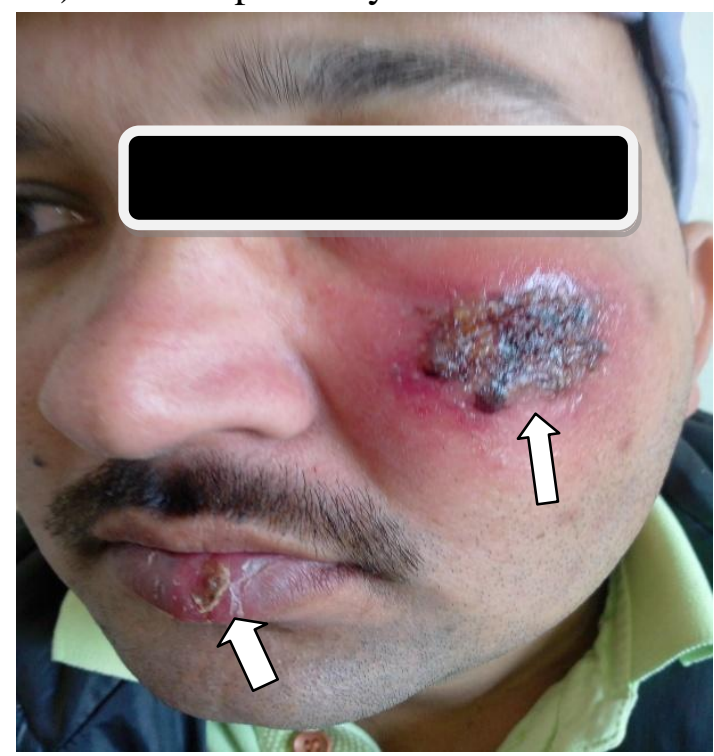

Figure 7: Plaques of Leishmaniasis over lower lip and cheek.

Lesions were distributed mainly over lips and oral commissures. Upper lip was most commonly affected in 43(58.9\%) cases followed by lower lip in $15(20.54 \%)$ cases, oral comissure in $9(12.33 \%)$ cases and both lips in $6(8.2 \%)$ cases. Out of these 73 cases, 21(28.76\%) patients had concurrent skin lesions of CL and 3(4.1\%) cases had involvement palatal, gingival and nasal mucosal involvement (MCL). Most cases were diagnosed on clinical basis but in 11 atypical cases biopsies and imprint smear examination were done. Out of these 11 cases, $8(72.7 \%)$ cases showed features suggestive papules, diffuse lip swelling resembling grannulomatous cheilitis and ulcers (Figure $7 \&$ 8).

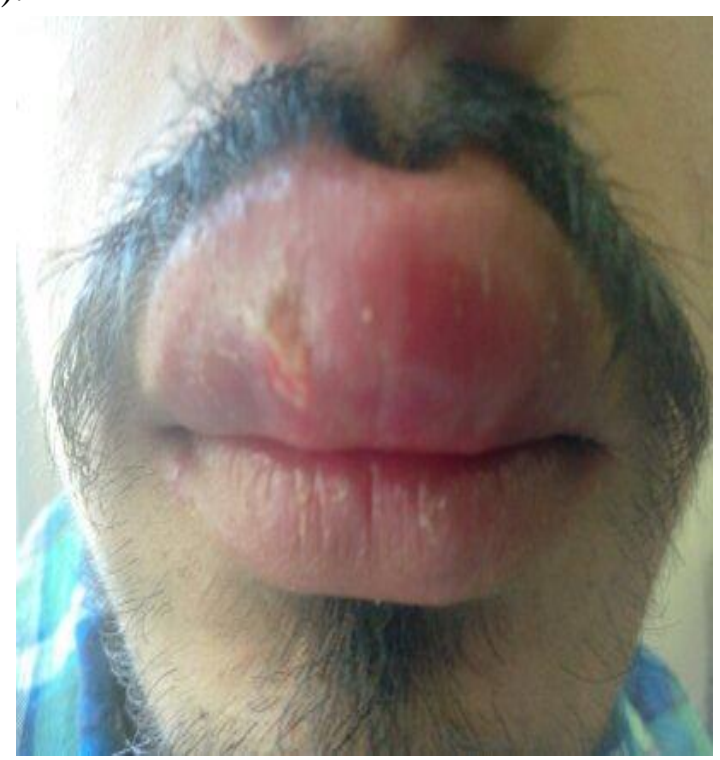

Figure 8: Diffuse upper lip swelling in a case of Lip Leishmaniasis.

of leishmaniasis on HPE and only 2(18.1\%) cases showed imprint smear positivity while in rest of the cases histopathology and imprint smears were non-conclusive.

Most [70(95.9\%)] cases were treated with 0.5-5 $\mathrm{ml}(100 \mathrm{mg} / \mathrm{ml})$ of SSG per lesion on three alternate days per month (one cycle). Only $3(4.1 \%)$ cases of MCL were treated with intravenous SSG in a dose of $20 \mathrm{mg} / \mathrm{kg}$ along with oral itraconazole $100 \mathrm{mg}$ BD doses. All cases responded well to treatment with few tolerable side effects. Most (94.5\%) of the cases achieved 
complete cure with three cycles of treatment, $3(4.1 \%)$ cases required 4 cycles and $1(1.36 \%)$ case required 5 cycles to have complete cure. Pain, swelling and bleeding from lips were minor side effects observed in all patients. Other less common side effects were lip ulceration in $41(56.16 \%)$ cases and reactive submandibular lymphadenopathy in $3(4.1 \%)$ cases.

\section{Discussion}

Leishmaniasis is amongst the top 5 diseases targeted by WHO under special research programme for tropical diseases. [7] Approximately 1.5 million new cases are affected each year and more than 350 million people are at risk of acquiring the disease. ${ }^{[7]}$ Leishmaniasis has variable clinical spectrum ranging from mildest cutaneous leishmaniasis (CL) to disfiguring mucocutaneous leishmaniasis (MCL) and most lethal type is known as Kala-Azar or visceral leishmaniasis (VL). ${ }^{[1]} \mathrm{CL}$ is caused by leishmania tropica complex in old world and by $\mathrm{L}$. braziliensis complex in the new world. ${ }^{[5]}$

CL may appear at unusual sites or present with atypical morphologies. Lesions at unusual sites are considered to be due to chance bites of sandflies at these sites. Disease at unexpected site with atypical morphology is probably due to altered host immune responses. ${ }^{[10]}$ The lip is not considered to be one of the common sites for CL. In literature, lip involvement is mostly described as part of MCL. Generally, MCL is a disease of new world and caused by L. braziliensis complex. ${ }^{[6]}$ Number of cases with LML has been reported from non-endemic areas of old world. In medical literature lip leishmaniasis is considered as a part of CL rather than of MCL. ${ }^{[7]}$ In 1997, Yaghoobi et al published a group of $17(.59 \%)$ patients with lip leishmaniasis from 2,861 patients with CL. ${ }^{\text {[11] }}$ Similarly CL localized to the lip has been reported from Saudi Arabia (16.7\%) ${ }^{[12]}$ and Turkey. ${ }^{[13]}$ Sajad et al reported 4 Cases of lip leishmaniasis from a Non endemic Region of Kashmir Valley of North India. ${ }^{[7]}$ Yeşilova et al reported 621 cases of lip leishmaniasis from Turkey. ${ }^{[14]}$
It is well established that L. donovani is known to cause kala-azar. In 10 to $20 \%$ of infections, there is reversal of the parasites from the viscerotrophic to the dermatotrophic form, which results in postkala-azar dermal leishmaniasis. ${ }^{[15]}$ In India, Thar deserts of Rajasthan, Punjab, Kerala and recently Himachal Pradesh are prevalent foci for CL. Here leishmania tropica has been the causative agent but recently, $L$. donovani has also been implicated. ${ }^{[16]} \mathrm{CL}$ due to $L$. donovani infection has already been reported from Kenya, Iraq, Shri Lanka and recently from Himachal Pradesh. ${ }^{[16]}$ Mohammadpour et al reported a case series from Iran and revealed that L. Tropica (causative agent for $\mathrm{CL}$ ) was detected from the lesions present over gingiva and lower lip (MCL). ${ }^{[1]}$ During this study we encountered three cases with oral and nasal mucosal involvement together with lip and cutaneous lesions. This reflects that either existing species (L. tropica or L. donovani) have tendency for mucosal infiltration or some different species is emerging in this region. Here the disease is not severe enough to cause mutilations, morbidities and mortalities. Most cases respond well to conventional intralesional antimonials (Inj.SSG). Various morphologies like furuncle-like, papular, nodular, chancriform or psoriasiform plaques have been described in literature. ${ }^{[2]}$ Similarly we found papules, nodules, plaques and diffuse lip swellings as common patterns in our patients. If lip lesions are classical or if typical CL lesions are present elsewhere on the skin then diagnosis is very simple. It is the diffuse lip swelling of one or both lips which often possesses diagnostic difficulty. Melkersson-Rosenthal syndrome, orofacial granulomatosis, cheilitis granulomatosa, Wegener granulomatosis oral, Crohn's disease, sarcoidosis, skin tuberculosis are various mimickers of lip leishmaniasis. ${ }^{[8]}$ In such cases clinical diagnosis of lip leishmaniasis remains a great a challenge with a significantly delayed or even an erroneous clinical diagnosis.

Identification of Leishmania parasites in dermal macrophages by skin biopsy or dermal scraping/imprint smear or culture can confirm the 
diagnosis. However, in chronic lesions, parasites may be scarce. Therefore, failure to visualize amastigotes on histopathology or imprint smears does not exclude the diagnosis of lip leishmaniasis. In such cases, culture and PCR are helpful diagnostic techniques but they are not unavailable everywhere. Patients with lip leishmaniasis are in good general health however, cosmetic disfiguring is of great concern.

\section{Conclusion}

In conclusion, this is the first study on of lip leishmaniasis reported from Himachal Pradesh. The disease doesn't seem to be uncommon but obviously under reported from this region. Although most patients had classical presentation so easily diagnosed but number of cases with unusual presentation posed diagnostic challenge. We recommend facilities of culture, PCR and molecular studies in order to know about the exact characteristics of parasite and vector in this region.

\section{References}

1. Mohammadpour I, Motazedian $\mathrm{MH}$, Handjani F, Hatam GR. Lip leishmaniasis: a case series with molecular identification and literature review. BMC Infectious Diseases 2017; 17:96

2. Bari AU, Rahman SB. Many faces of Cutaneous Leishmaniasis. Indian $\mathbf{J}$ Dermatol Venereol Leprol 2008;74:23-27. [PubMed]

3. Veraldi S, Rigoni C, Gianotti R. Leishmaniasis of the lip. Acta Derm Venereol. 2002; 82:469-70. [PubMed]

4. Motta ACF, Lopes MA, Ito FA, CarlosBregni R, De Almeida OP, Roselino AM. Oral leishmaniasis: a clinicopathological study of 11 cases. Oral Dis. 2007;13:335040.

5. Torres-Guerrero E, Quintanilla-Cedillo MR, Ruiz-Esmenjaud J, Arenas R. Leishmaniasis: a review [version 1; referees: 2 approved] F1000Research
2017, 6(F1000 Faculty Rev):750 (doi: 10.12688/f1000research.11120.1)

6. Kharfi M, Fazaa B, Chaker E, Kamoun MR. Mucosal localization of leishmaniasis in Tunisia: 5 cases. Ann Dermatol Venereol 2003;130(1 Pt 1):27-30.

7. Sajad P, Rather S, Hassan I, Qureshi W. Primary Lip Leishmaniasis-Report of 4 Cases from a Non-endemic Region of Kashmir Valley of North India and Review of Literature. JAMMR 2017;24(1):1-5.

8. Ekiz Ö, Rifaioglu EN, Sen BB, Çulha G, Özgür T, Dogramaci AÇ. Leishmaniasis Recidiva Cutis of the Lips Mimicking Granulomatous Cheilitis. Indian J Dermatol 2015;60:216.

9. Bari A. Epidemiology of cutaneous leishmaniasis. Journal of Pakistan Association of Dermatologists.2006; 16:156-162.

10. Veraldi S, Rigoni C, Gianotti R. Leishmaniasis of the lip. Acta Derm Venereol. 2002; 82: 469-70. [PubMed]

11. Yaghoobi R, Hoghooghi-Rad N. Lip leishmaniasis in the southwest of Iran during the last decade. Iranian $\mathbf{J}$ Dermatol.1997;19-23.

12. Al-Tawfiq JA, Abu Khamsin A. Cutaneous leishmaniasis: A 46-year study of the epidemiology and clinical features in Saudi Arabia (1956-2002). Int J Infect Dis. 2004;8(4):244-50.

13. Ok UZ, Balcioğlu IC, Taylan Ozkan A, Ozensoy S, Ozbel Y. Leishmaniasis in Turkey. Acta Trop. 2002;84(1):43-8.

14. Yesilova Y, Aksoy M, Surucu HA, Uluat A, Ardic N, Yesilova A. Lip leishmaniasis: Clinical characteristics of 621 patients. Int J Crit Illn Inj Sci 2015;5:265-6.

15. Salotra P, Sreenivas G, Pogue GP, et al. Development of a species-specific PCR assay for detection of Leishmania donovani in clinical samples from patients with kala-azar and post-kalaazar dermal 
leishmaniasis. J Clin Microbiol 2001;39:849-54.

16. Sharma NL, Mahajan VK, Kanga A, Sood A, Katoch VM, Mauricio I, et al. Localized Cutaneous Leishmaniasis due to Leishmania Donovani And Leishmania Tropica: Preliminary Findings of the Study of 161 New Cases From A New Endemic Focus In Himachal Pradesh, India. Am J Trop Med Hyg 2005;72(6):819-24. 